\title{
Magnetic resonance imaging of the coronary arteries: clinical results from three dimensional evaluation of a respiratory gated technique
} R J M van Geuns, H G de Bruin, B J W M Rensing, P A Wielopolski, M D Hulshoff,
P M A van Ooijen, M Oudkerk, P J de Feyter

\begin{abstract}
Background-Magnetic resonance coronary angiography is challenging because of the motion of the vessels during cardiac contraction and respiration. Additional challenges are the small calibre of the arteries and their complex three dimensional course. Respiratory gating, turboflash acquisition, and volume rendering techniques may meet the necessary requirements for appropriate visualisation. Objective-To determine the diagnostic accuracy of respiratory gated magnetic resonance imaging (MRI) for the detection of significant coronary artery stenoses evaluated with three dimensional postprocessing software.

Methods-32 patients referred for elective coronary angiography were studied with a retrospective respiratory gated three dimensional gradient echo MRI technique. Resolution was $1.9 \times 1.25 \times 2 \mathrm{~mm}$. After manual segmentation three dimensional evaluation was performed with a volume rendering technique.

Results-Overall $74 \%$ (range $50 \%$ to $90 \%$ ) of the proximal and mid coronary artery segments were visualised with an image quality suitable for further analysis. Sensitivity and specificity for the detection of significant stenoses were $50 \%$ and $91 \%$, respectively.

Conclusions-Volume rendering of respiratory gated MRI techniques allows adequate visualisation of the coronary arteries in patients with a regular breathing pattern. Significant lesions in the major coronary artery branches can be identified with a moderate sensitivity and a high specificity.

(Heart 1999;82:515-519)
\end{abstract}

Keywords: magnetic resonance imaging; coronary artery disease; coronary angiography; computer assisted image processing

Magnetic resonance imaging (MRI) is a truly non-invasive technique which is not associated with radiation and is nowadays available for clinical use. MRI of the coronary arteries is, however, a challenging task owing to motion of the vessels during cardiac contraction and respiration, the complexity of the anatomy in three dimensions, the small calibre of the vessels, and the fact that the vessels are embedded in fat which produces a competing signal.
Coronary artery motion during cardiac contraction is successfully minimised by ECG triggering, with data collection over 100 to 150 ms during mid to late diastole. Reduction of respiratory motion is achieved with breath holding or with respiratory gated techniques. The complex course of the coronary anatomy can be evaluated with two dimensional (2D) or preferably three dimensional (3D) acquisition techniques.

MRI of the coronary arteries (MRCA) was first performed in 1993 with a single slice breathhold technique (2D-MRCA). ${ }^{1-3} \mathrm{Al}-$ though initial results seemed encouraging, the use of 2D-MRCA is limited by its complex setup for image orientation and its dependency on consistent breath holding. ${ }^{4}$ The use of a respiratory gated technique (navigator) for $\mathrm{MRCA}^{67}$ was introduced later as another possibility to reduce respiratory blur. Without restrictions in imaging time imposed by the patient's breathhold limits, longer imaging sequences can be used. This allows the complex coronary artery anatomy to be studied with a three dimensional technique (3DMRCA).

Evaluation of a 3D-MRCA dataset can be performed with multiplanar reformatting techniques, ${ }^{89}$ producing slices in any desired plane through the volume. However, this technique is limited because it does not use all the information present in a three dimensional dataset. This can be overcome by using the volume rendering technique ${ }^{10}$ present in special three dimensional viewing software, which uses all the information in a three dimensional dataset.

In this study we determined the diagnostic accuracy of respiratory gated 3D-MRCA for the detection of coronary artery stenoses evaluated with a volume rendering technique.

\section{Methods}

PATIENTS

The study population consisted of 32 patients (20 men, 12 women; age 32 to 73 years) who were referred for elective coronary angiography. Exclusion criteria were previous coronary bypass operation, intracoronary stent implantation, artificial pacemaker, intracranial clips, claustrophobia, and non-sinus rhythm. The protocol was approved by our hospital committee on medical ethics and clinical investigation.
Accepted for publication 9 April 1999 


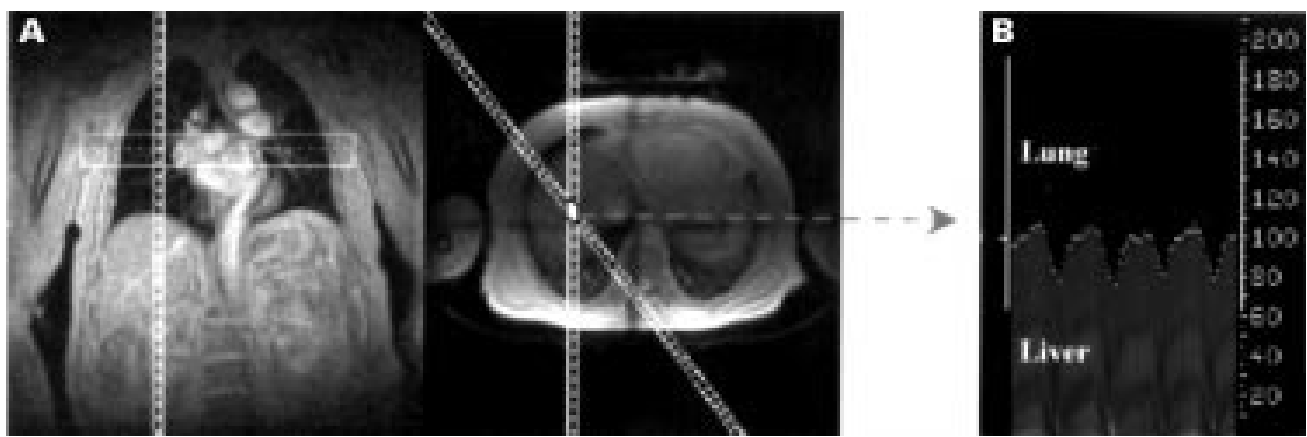

Figure 1 Retrospective respiratory gated magnetic resonance imaging of the coronary arteries. (A) Respiratory motion is determined by two excitation bands that intersected at the dome of the right hemidiaphragm. (B) Respiration pattern during 30 seconds. The diaphragm position is determined for each acquisition window. Retrospectively only data from end expiration are selected for image reconstruction.

MAGNETIC RESONANCE IMAGING

Subjects were studied in a supine position, with a four channel quadrature body phased array coil placed over the thorax, in a $1.5 \mathrm{~T}$ whole body magnetic resonance imaging system (Vision; Siemens, Erlangen, Germany). Coronary artery imaging was performed using a standard Siemens three dimensional gradient
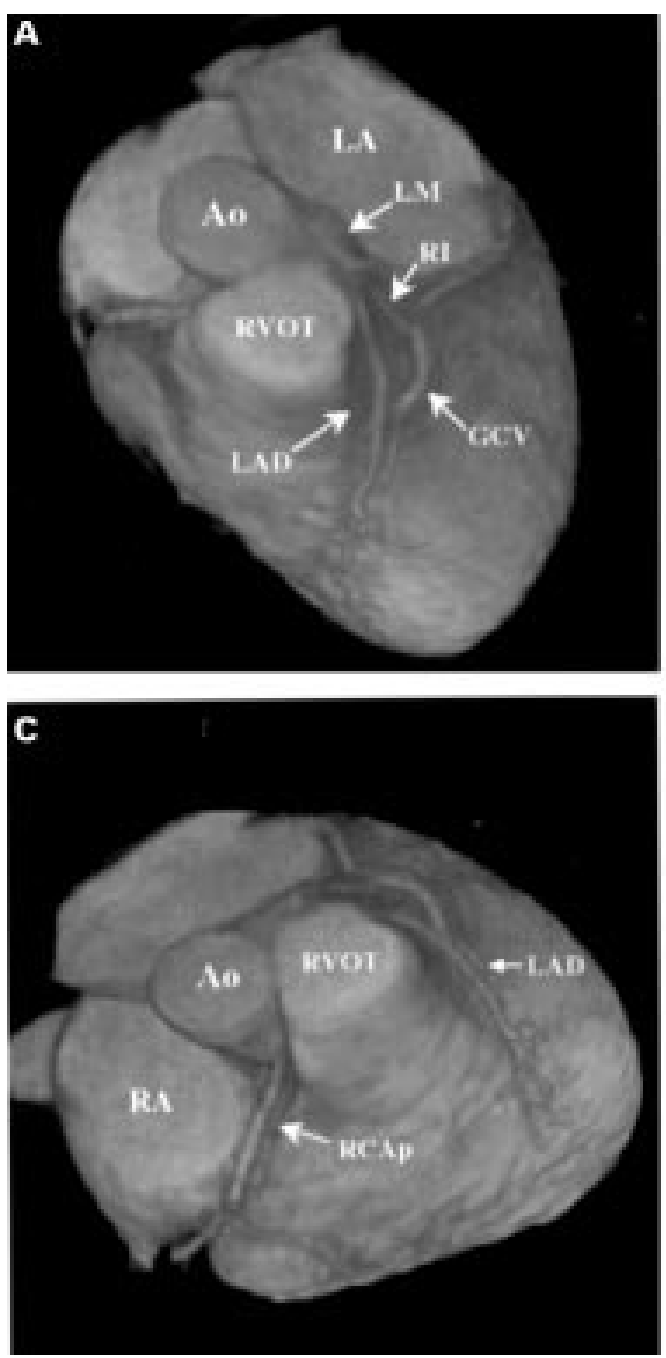

echo sequence with retrospective respiratory gated technique described by $\mathrm{Li}$ et $a .^{7} \mathrm{~A}$ chemical shift fat suppression pulse was used to suppress the signal from the epicardial fat surrounding the coronary arteries. In our setup we used three slabs of $32 \mathrm{~mm}$ thickness with a $25 \%$ overlap; section thickness was $2 \mathrm{~mm}$. The matrix size was $128 \times 256$ with a rectangular
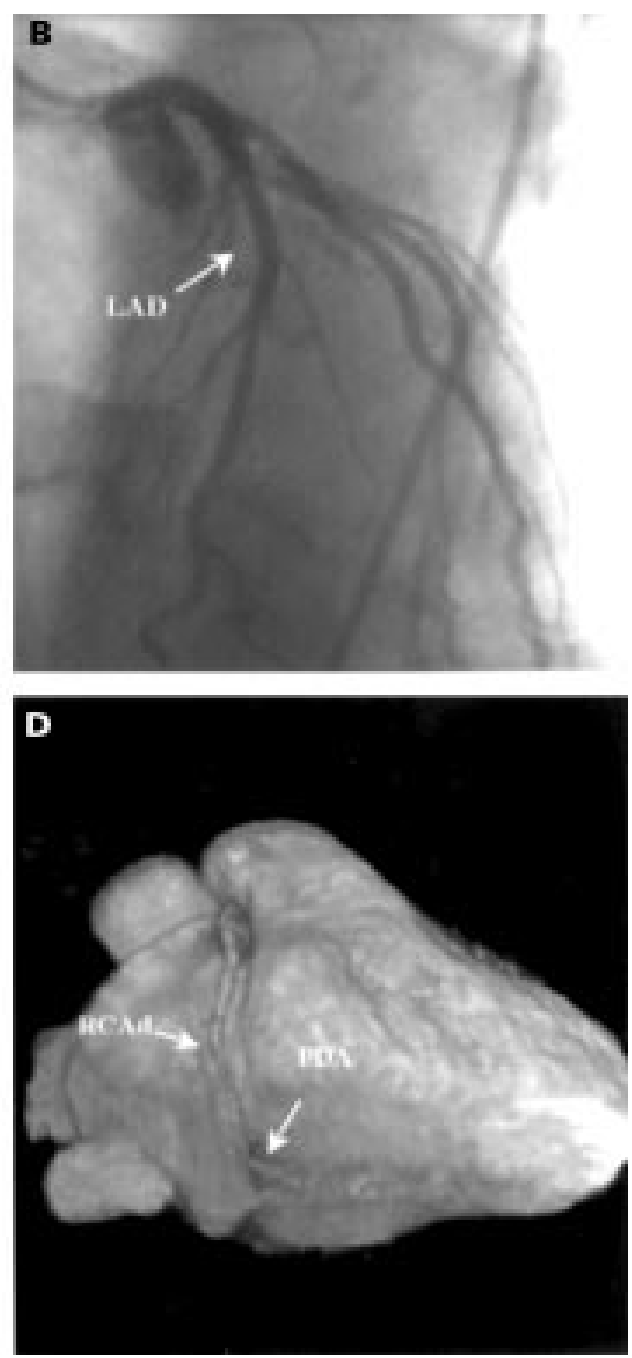

Figure 2 Volume rendering of a patient without significant stenoses. (A) Cranial view of the left anterior descending coronary artery (LAD). The great cardiac vein (GCV) overlaps intermediate branches (RI), which hampers evaluation of this segment. (B) Corresponding conventional selective coronary angiogram. (C) Rotation to right cranial view for proximal right coronary artery (RCAp) between right ventricular outflow tract (RVOT) and right atrium (RA). The right auricle is manually removed from the data. (D) Rotation to right caudal view for distal right coronary artery (RCAd) and origin of posterior descending artery $(P D A)$. Ao, aorta; $L A$, left atrium. 
Table 1 Accessibility of different coronary artery segments by magnetic resonance coronary angiography

\begin{tabular}{lc}
\hline & $M R I$ \\
\hline RCA-proximal part & $93 \%$ \\
RCA-middle part & $76 \%$ \\
LM & $97 \%$ \\
LAD-proximal part & $90 \%$ \\
LAD-middle part & $76 \%$ \\
LCx-proximal part & $76 \%$ \\
LCx-middle part & $28 \%$ \\
\hline
\end{tabular}

LAD, left anterior descending coronary artery; LCx, left circumflex coronary artery; LM, left main coronary artery; RCA, right coronary artery.

field of view of $240 \times 320 \mathrm{~mm}$, resulting in an inplane resolution of $1.9 \times 1.25 \mathrm{~mm}$. The time of repetition (TR) was $7.4 \mathrm{~ms}$, the time of echo (TE) was $2.7 \mathrm{~ms}$, and the flip angle varied from $20^{\circ}$ to $90^{\circ}$. The acquisition window $(128 \mathrm{~ms})$ was set for mid to late diastole. Each slab was acquired in eight to 12 minutes, depending on the heart rate.

Retrospective respiratory gating was performed by a navigator echo created with two excitation bands placed to intersect at the dome of the right hemidiaphragm (fig 1). Together, these two bands measure the diaphragmatic position before data acquisition. The most common position of the diaphragm is determined and chosen as the gating centre. Commonly this is end expiration. Each line of data was acquired five times to ensure complete sampling of the respiratory excursion. Data within a range of $\pm 1 \mathrm{~mm}$ from the gating centre are used for image reconstruction. If no acquisitions of a certain data line are within the acceptance range, the acquisition obtained at the diaphragm displacements closest to the gating centre are used at image reconstruction.

The total examination time for MRI of each subject, including positioning of the patient, scout imaging, and setting up the navigator, was approximately one hour.

CONVENTIONAL CORONARY ANGIOGRAPHY All subjects underwent standard selective coronary artery angiography within one month of the magnetic resonance examination. Angiography was performed using the Judkins
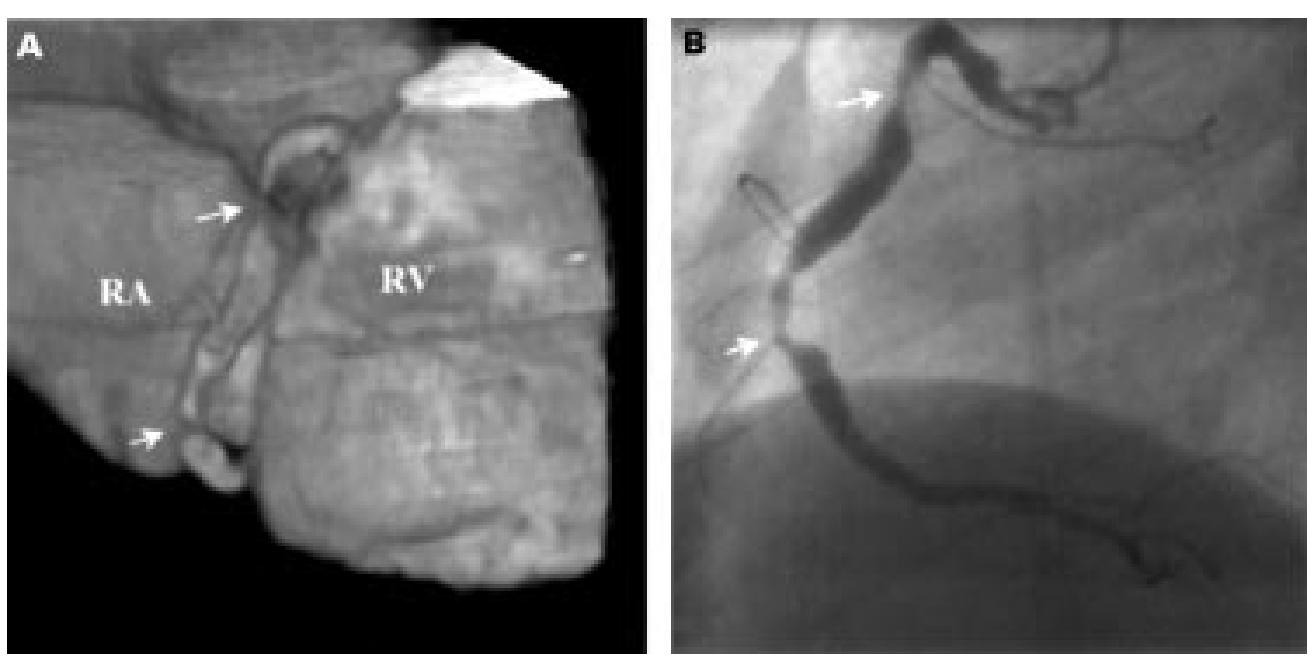

Figure 3 (A) Magnetic resonance imaging. Detailed view on the right coronary artery in the atrioventricular groove between the right ventricle $(R V)$ and atrium $(R A)$. The arrows indicate stenoses in the proximal and mid segment. (B) Corresponding conventional coronary angiogram. technique. ${ }^{11}$ The selective angiograms were jointly interpreted by two experienced cardiologists not familiar with the MRI results. The coronary tree was divided into proximal and mid segments according to AHA guidelines. ${ }^{12}$ These segments were graded as either no significant disease $(<50 \%$ diameter stenosis) or significant disease $(>50 \%$ diameter stenosis). In case of disagreement a final decision was made by a third cardiologist.

INTERPRETATION OF MAGNETIC RESONANCE CORONARY ANGIOGRAMS

The magnetic resonance datasets were transferred to a stand alone workstation (MagicView; Siemens, Erlangen, Germany). By manual segmentation, the chest wall, lung vessels, and overlapping parts of the left and right auricle were removed from the dataset. Manual segmentation required 20 to 30 minutes for 60 slices. After image segmentation the datasets were transferred to a dedicated graphic workstation (Indigo2; Silicon Graphics, Mountain View, California, USA) for three dimensional evaluation with a volume rendering technique $e^{13-15}$ using commercially available software (VoxelView; Vital Images Inc, Minneapolis, Minnesota, USA). In the volume rendering technique, all image pixels are integrated to project a three dimensional dataset as a single image. For this a certain opacity is assigned to each pixel, based on its value in the dataset. A projection method will pass through all the pixels from back to front and calculates a value to display on the screen. The opacity for certain structures will improve the three dimensional impression of the image. The datasets can be rotated in every direction for optimal visualisation of the major coronary artery branches (fig 2). The three dimensional reconstructions together with the original axial slices were reviewed independently by a radiologist and a cardiologist. The left main coronary artery, proximal and mid right coronary artery, left anterior descending coronary artery, and circumflex artery were graded as assessable, non-assessable, or outside the acquired volume. The assessable segments 

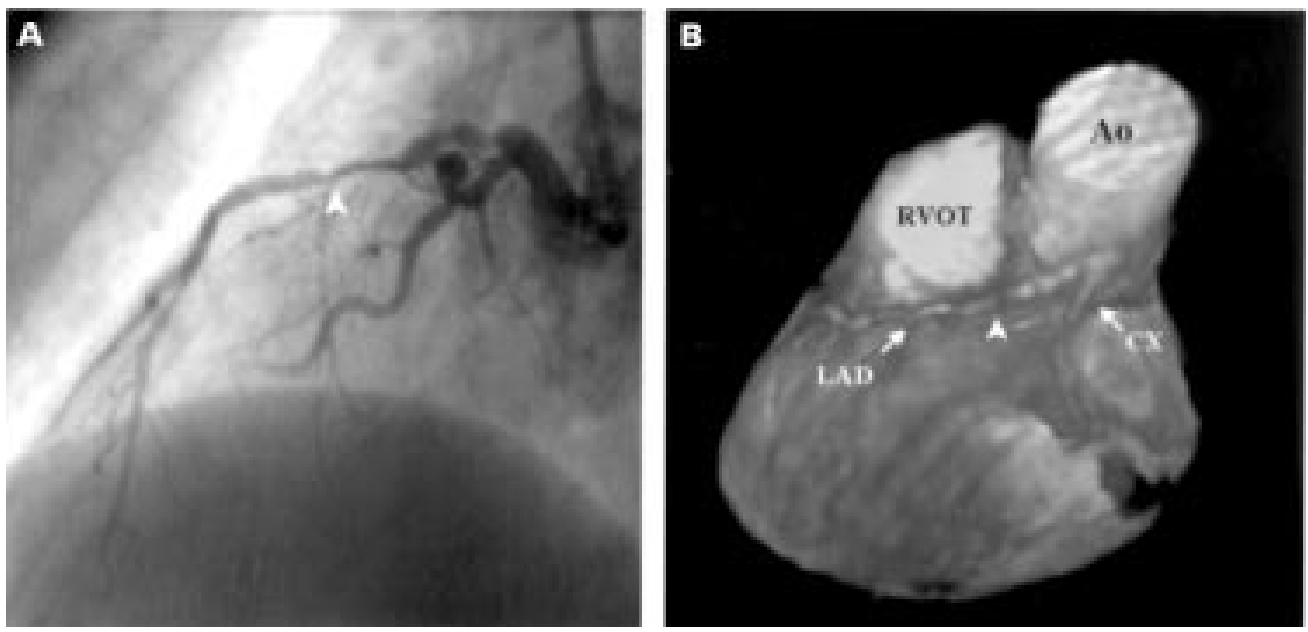

Figure 4 Example of a significant stenosis (arrow head) in the left anterior descending coronary artery (LAD).

(A) Conventional coronary angiogram. (B) Magnetic resonance imaging. Ao, aorta; LM, left main; LV, left ventricle; RVOT, right ventricular outflow tract.

were graded as either no significant or significant disease. In case of disagreement a third investigator made a final decision.

\section{STATISTICS}

A selective coronary angiogram served as the gold standard for determining the diagnostic value of the non-invasive coronary angiogram. The diagnostic accuracy of magnetic resonance coronary angiography for detecting significant stenoses in a segment is expressed as sensitivity, specificity, and positive and negative predictive value. The diagnostic value for the presence of significant coronary artery disease was also calculated on a per patient basis.

\section{Results}

Of the 32 studies, three were not completed owing to ECG triggering problems, technical failure, or unknown claustrophobia. The mean interval between the examinations was 15 days. Overall $151(74 \%)$ of the 203 coronary artery segments were assessable by MRCA, ranging from $97 \%$ for the left main coronary artery to $28 \%$ for the mid-circumflex coronary artery (table 1). In these segments 26 significant lesions were present (left main and left anterior descending, 11; right coronary, 11; circumflex, 4). An example of two consecutive stenoses in the right coronary artery is shown in fig 3; an example of a stenosis in the left anterior descending coronary artery is shown in fig 4 . The sensitivity and specificity for the detection of a stenosis in a segment were $50 \%$ and $91 \%$, respectively. The diagnostic accuracy for the individual vessels is summarised in table 2 . The

Table 2 Diagnostic accuracy for the detection of significant coronary artery stenosis by magnetic resonance coronary angiography

\begin{tabular}{llllll}
\hline & Total & LM LAD & $L C x$ & RCA & Patient \\
\hline Sensitivity & $50 \%$ & $55 \%$ & $50 \%$ & $45 \%$ & $76 \%$ \\
Specificity & $91 \%$ & $92 \%$ & $95 \%$ & $87 \%$ & $73 \%$ \\
PPV & $54 \%$ & $55 \%$ & $67 \%$ & $50 \%$ & $81 \%$ \\
NPV & $90 \%$ & $92 \%$ & $91 \%$ & $85 \%$ & $67 \%$ \\
\hline
\end{tabular}

$\mathrm{LAD}$, left anterior descending coronary artery; $\mathrm{LCx}$, left circumflex coronary artery; LM, left main coronary artery; NPV, negative predictive value; PPV, positive predictive value; RCA, right coronary artery. sensitivity for selection of patients with any significant disease was $76 \%$, with a specificity of $73 \%$.

\section{Discussion}

In this study we used a standard technique available on a modern magnetic resonance scanner. The use of a single navigator signal from the diaphragm increases the image quality of MRCA. ${ }^{16}$ The results reported by other investigators using this technique for the detection of coronary artery stenosis differ widely, ranging from inadequate to reasonably accurate. ${ }^{817} 18$ Here we showed high specificity but only moderate sensitivity, owing to insufficient image quality. The major reason for poor image quality is residual respiratory blur originating from irregular respiration patterns, ${ }^{19}$ with data acquisition outside the desired gating window. ${ }^{18}{ }^{20}$ In general only $25-30 \%$ of the data are acquired within the gating window. False negative MRCA interpretations are caused by retrograde flow distal to complete occlusions and volume averaging of vessels with adjacent structures, ${ }^{21}$ or by the inability to distinguish coronary arteries from veins. ${ }^{5}$ False positive interpretations arise from the low contrast between the coronary arteries and their surrounding tissue, motion artefacts, ${ }^{20}$ or errors during manual segmentation.

A negative test result from a test with a sensitivity of only $50 \%$ unfortunately does not rule out the undetected presence of significant coronary artery disease. This will limit the use of this technique as a clinical screening method. This may be even more problematic in a group of patients with a lower prevalence of disease, compared with our patients who were referred for elective coronary angiography.

Improvement in image quality of respiratory gated MRCA will reduce false interpretations. This can be achieved by correlating image position with respiratory motion. ${ }^{22}$ The acquisition volume is shifted caudally over a certain distance during inspiration so that the percentage of data within the gating window increases. The shifted distance is different for each 
coronary artery and has to be determined for every patient individually. So far clinical applicability has not been reported. Alternatively MRCA can be performed with new breath holding techniques, such as volume coronary angiography using targeted scans (VCATS), that acquire targeted volumes along the coronary arteries. ${ }^{23}$ Respiratory blur is minimised, and acquisition time is reduced to less than 30 minutes.

The use of bolus injections of contrast agents, given over a 20 second period, has dramatically improved magnetic resonance angiography of peripheral arteries. ${ }^{24}{ }^{25}$ GadoliniumDTPA, the contrast agent most often used, diffuses rapidly extravascularly and it is therefore not possible to maintain a high intracoronary concentration during 30 to 40 minutes after intravenous injection. Intravascular magnetic resonance contrast agents may be an alternative. Both respiratory gated and breath hold techniques are expected to benefit from intravascular contrast agents. ${ }^{26}{ }^{27}$ The resulting increase of contrast to noise ratio will improve visualisation of the coronary arteries and allow the use of high resolution techniques. Unfortunately intravascular contrast agents are presently in a preclinical phase and registration may still take several years.

Volume rendering as a technique for evaluation of three dimensional datasets on a two dimensional surface has proved valuable in magnetic resonance and computed tomographic angiography of both central and peripheral arteries. ${ }^{28}{ }^{29}$ Recently the same technique has been introduced in the evaluation of electron beam tomography. ${ }^{30}$ Its main advantage is the nearly anatomical images produced, with the possibility of viewing the data from different angles to obtain optimal orientation for individual coronary arteries. The high computational power necessary for this technique has limited its use hitherto but with the present commercially available hardware and software volume rendering can now be performed with reasonable speed.

\section{CONCLUSIONS}

Respiratory gated MRCA is a technique with an uncomplicated setup that can be performed in a clinical setting. At this stage of development image quality is sufficient in only $70 \%$ of patients. In patients with a regular breathing pattern significant lesions in the major coronary artery branches can be identified with moderate sensitivity and high specificity. Volume rendering of respiratory MRCA creates highly interpretable images, but improvements in the magnetic resonance technique are necessary before it becomes a clinically reliable tool.

1 Manning WJ, Li W, Boyle NG, et al. Fat-suppressed breathhold magnetic resonance coronary angiography. Circulation 1993;87:94-104

2 Pennell DJ, Keegan J, Firmin DN, et al. Magnetic resonance imaging of coronary arteries: technique and preliminary results. Br Heart $\mathcal{F}$ 1993;70:315-26.
3 Duerinckx AJ, Urman MK. Two-dimensional coronary MR angiography: analysis of initial clinical results. Radiology 1994;193:731-8

4 van Rossum AC, Post JC, Visser CA. Coronary imaging using MRI. Herz 1996;21:97-105.

5 Duerinckx AJ, Atkinson DP, Mintorovitch J, et al. Two-dimensional coronary MRA: limitations and artifacts. Eur Radiol 1996;6:312-25.

6 Hofman MB, Paschal CB, Li D, et al. MRI of coronary arteries: $2 \mathrm{D}$ breath-hold vs $3 \mathrm{D}$ respiratory-gated acquisition. 7 Comput Assist Tomogr 1995;19:56-62.

7 Li D, Kaushikkar S, Haacke EM, et al. Coronary arteries: three-dimensional MR imaging with retrospective respiratory gating. Radiology 1996;201:857-63.

8 Kessler W, Achenbach S, Moshage W, et al. Usefulness of respiratory gated magnetic resonance coronary angiography in assessing narrowings $>$ or $=50 \%$ in diameter in native coronary arteries and in aortocoronary bypass native coronary arteries and in aortoco

9 Post JC, van Rossum AC, Hofman MB, et al. Protocol for two-dimensional magnetic resonance coronary angiography studied in three-dimensional magnetic resonance data sets. Am Heart f 1995;130:167-73.

10 Drebin R, Carpentier L, Hanrahan P. Volume rendering. Comput Graphics 1988;22:65-74.

11 Judkins MP. Selective coronary arteriography. I. A percutaneous transfemoral technic. Radiology 1967;89:815-24.

12 Austen WG, Edwards JE, Frye RL, et al. A reporting system on patients evaluated for coronary artery disease. Report of the Ad Hoc Committee for Grading of Coronary Artery Disease, Council on Cardiovascular Surgery, American Heart Association. Circulation 1975;51(suppl):5-40.

13 Ney D, Fishman E, Magid D. Volumetric rendering of computed tomography data: principles and techniques. Comput Graphics Applic 1990;10:24-32.

14 Johnson PT, Heath DG, Kuszyk BS, et al. CT angiography with volume rendering: advantages and applications in splanchnic vascular imaging. Radiology 1996;200:564-8.

15 Fishman EK, Magid D, Ney DR, et al. Three-dimensional imaging. Radiology 1991;181:321-37.

16 Wang Y, Rossman PJ, Grimm RC, et al. Navigator-echobased real-time respiratory gating and triggering for reduction of respiration effects in three-dimensional coronary MR angiography. Radiology 1996;198:55-60.

17 Post JC, van Rossum AC, Hofman MB, et al. Threedimensional respiratory-gated MR angiography of coronary arteries: comparison with conventional coronary angiography. Am f Roentgenol 1996;166:1399-404.

18 Muller MF, Fleisch M, Kroeker R, et al. Proximal coronary artery stenosis: three-dimensional MRI with fat saturation and navigator echo. 7 Magn Reson Imaging 1997:7:644-51.

19 Taylor AM, Jhooti P, Wiesmann F, et al. MR navigator-echo monitoring of temporal changes in diaphragm position:
implications for MR coronary angiography. $\mathcal{F}$ Magn Reson implications for MR cor

20 Achenbach S, Kessler W, Moshage WE, et al. Visualization of the coronary arteries in three-dimensional reconstructions using respiratory gated magnetic resonance imaging. Coron Artery Dis 1997;8:441-8.

21 Woodard PK, Li D, Haacke EM, et al. Detection of coronary stenoses on source and projection images using three-dimensional MR angiography with retrospective respiratory gating: preliminary experience. Am f Roentgenol piratory gating: p

22 Danias PG, McConnell MV, Khasgiwala VC, et al. Prospective navigator correction of image position for coronary MR angiography. Radiology 1997;203:733-6.

23 Wielopolski $\mathrm{P}$, vanGeuns R, deFeyter P, et al. Breath-hold coronary $\mathrm{MR}$ angiography with volume targeted imaging. Radiology 1998;209:209-19.

24 Leung DA, McKinnon GC, Davis CP, et al. Breath-hold, contrast-enhanced, three-dimensional MR angiography. Radiology 1996;200:569-71.

25 Prince MR, Narasimham DL, Stanley JC, et al. Breath-hold gadolinium-enhanced MR angiography of the abdominal aorta and its major branches. Radiology 1995;197:785-92.

26 Lauffer RB, Parmelee DJ, Dunham SU, et al. MS-325: albumin-targeted contrast agent for MR angiography. Radiology 1998;207:529-38.

$27 \mathrm{Li} \mathrm{D}$, Zheng J, Weinmann $\mathrm{H}$, et al. Comparison of intravascular and extravascular contrast agents in coronary artery imaging [abstract]. Proceedings of the sixth meeting of the International Society for Magnetic Resonance in Medicine. Sydney, 1998.

28 Johnson PT, Heath DG, Kuszyk BS, et al. CT angiography: thoracic vascular imaging with interactive volume rendering technique. $\mathcal{F}$ Comput Assist Tomogr 1997;21:110-14.

29 Kuszyk BS, Heath DG, Ney DR, et al. CT angiography with volume rendering: imaging findings. Am f Roentgenol 1995; 165:445-8.

30 Rensing BJ, Bongaerts A, van Geuns RJ, et al. Intravenous coronary angiography by electron beam computed tomography: a clinical evaluation. Circulation 1998;98: 2509-12. 